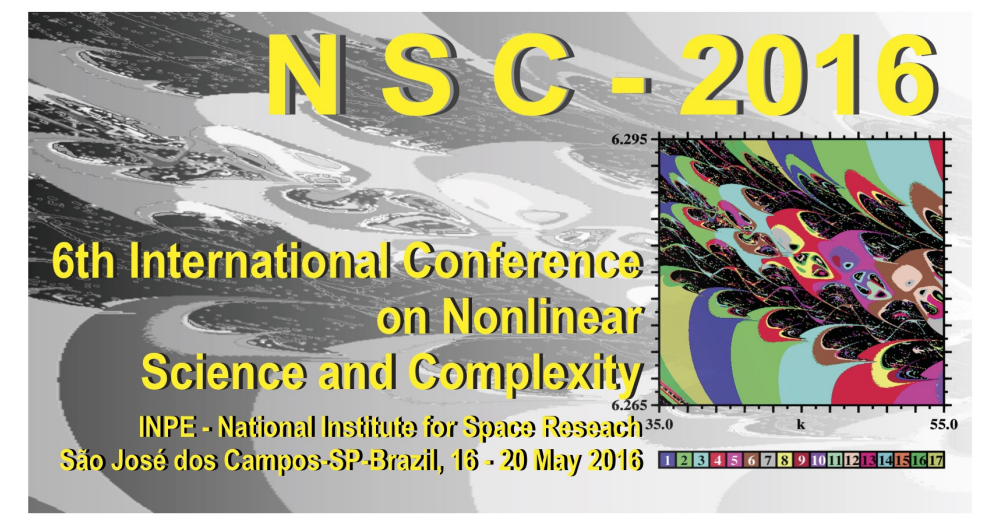

INPE - National Institute for Space Research

São José dos Campos - SP - Brazil - May 16-20, 2016

\title{
REACTIVE MODEL FOR CONVERGENCE OF ACTIVE AGENTS TO MOVING FORMATIONS
}

\author{
Vander L. S. Freitas ${ }^{1}$ and Elbert E. N. Macau ${ }^{2}$ \\ ${ }^{1} \mathrm{PhD}$ candidate on Applied Computing at INPE, São José dos Campos, Brazil, vander.freitas@inpe.br \\ ${ }^{2}$ Associated Laboratory for Computing and Applied Mathematics (LAC) - INPE, São José dos Campos, Brazil, elbert.macau@inpe.br
}

\begin{abstract}
Collective motion emerges from interactions among individuals as observed in nature with flocks of birds. In this work, we elaborated a reactive model to lead groups of autonomous mobile agents to moving formations, starting from random positions. In this model, the agents interact via repulsion, alignment and attraction rules, and do not keep memory of previous interactions. The result is a parallel formation of agents moving in a desired direction.
\end{abstract}

keywords: Nonlinear Dynamics and Complex Systems, Control in Complex Systems, Synchronization in Nonlinear Systems, Collective Motion, Multiagent Systems.

\section{INTRODUCTION}

Nature exhibits many emergent motions in collections of living beings. These global behaviours are the result of local interactions among the agents. Common situations of the emergence of collective motion are in predator escaping, food search or hunting, for example.

Collective motion is a phenomenon that occurs in collections of agents, similar or not, who interact with each other, resulting in ordered motion [1]. Those interactions can be among close neighbours or in the context of a more evolved interaction among the agents. Collective motion is present everywhere, from colonies of bacteria to schools of fish [2].

The motivation for the study of collective motion is to understand the interaction rules among the units. These rules may be applied to artificial agents so they can work collaboratively in some tasks. Then it is possible to create a link between control theory and applications of collective motion like groups of robots, unnamed aerial vehicles, unnamed underwater vehicle or even satellites.

In this work we elaborated a reactive model for convergence of active agents to moving formations. The agents interact via rules of repulsion, alignment and attraction. The individuals begin at random positions inside an area and after a transient of interactions they converge to a moving formation heading to a predefined direction of motion.

\section{REACTIVE MODEL}

Model components (Figure 1):

- Virtual agent: Its role is to point out the desired motion direction and the region in which we want the agents to converge to a parallel formation. It is not a real agent of the model.

- Virtual agent interaction radius (VAIR): Circular region centered in virtual agent.

- Reactive agents: The agents of the model. The main characteristic is that they do not keep memory of previous interactions, i.e., they act according to the current system state.

The aim is to make the agents to enter the VAIR and follow the virtual agent in a parallel formation. For this purpose they interact via adjustment in their velocity module and rules (interaction strengths) based on repulsion, alignment and attraction. We call these interaction rules as "virtual forces". Here the concept of force is related to how the 


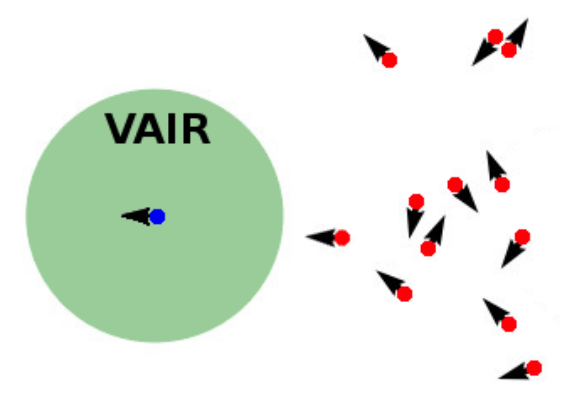

Figure 1 - Model components: Virtual agent is in blue, agents are in red and VAIR is in green.

presence of the neighbors of an agent can impose changes on its heading angle.

The velocity module adjustment is done according to the position of the agent in relation to the VAIR. When an agent is outside the VAIR its velocity module is higher than when it is inside. When an agent is entering the VAIR it suffers a deceleration, according to Eq 1 .

$$
\left|v_{i}\right|=\left|v_{a}\right|-\frac{\left(\left|v_{i n i}\right|-\left|v_{a}\right|\right)\left(t-t_{f}\right)}{\Delta t_{i}}
$$

so that $v_{i}$ is the velocity of agent $i, v_{a}$ is the velocity of the virtual agent, $v_{i n i}$ is the velocity of agent $i$ at the moment it started entering the VAIR, $t$ is the current time, $t_{f}$ is the time instant in which the acceleration will finish and $\Delta t_{i}$ is the total time of this procedure.

In this equation $\Delta t_{i}=\bar{t}+r n d$, in which $\bar{t}$ represents the approximate time for the agent to move from the VAIR boundary to the virtual agent's position, and $r n d$ is a random number in the interval $r n d \in(-\gamma, \gamma)$. Here we used $\gamma \doteq \bar{t}$ to guarantee a homogeneous distribution of agents inside the VAIR.

When an agent is leaving the VAIR its velocity module increases according to $\mathrm{Eq} 2$. In this case, $\left|v_{\text {out }}\right|$ represents the maximum velocity module outside the VAIR.

$$
\left|v_{i}\right|=\left|v_{a}\right|-\frac{\left(\left|v_{\text {out }}\right|-\left|v_{\text {ini }}\right|\right)\left(t-t_{f}\right)}{\Delta t_{i}}
$$

We used five interaction rules to achieve the parallel formation in the desired direction of motion. The rules are written in the form of forces, as follows (Figure 2):

- $F_{a}$ (Alignment): Orders the agent to align with the average heading angles of its neighbors.

- $F_{c}$ (Cohesion): Orders the agent to go to the center of mass of its neighbors.

- $F_{a v}$ (Alignment with virtual agent): Orders the agent to align with the virtual agent heading angle.

- $F_{c v}$ (Cohesion with virtual agent): Orders the agent to move towards the virtual agent.

- $F_{s}$ (Separation): Orders the agent to move apart from its nearest neighbor.
All of those forces are applied to the agent through heading angle adjustment. They act directly on the heading angle of the agents at discrete time. Each time unity $(t u)$ corresponds to a travel of $1 \mathrm{bl}$ (body length of an agent) of virtual agent, i.e., $\left|v_{a}\right|=1 \mathrm{bl} / \mathrm{tu}$.

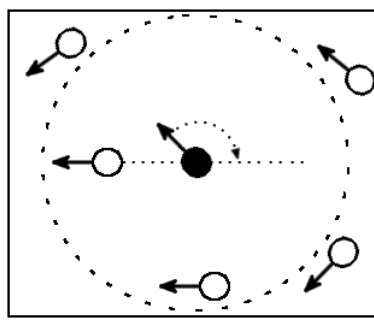

(a) $F_{s}$

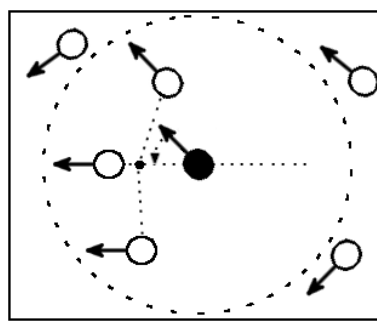

(c) $F_{c}$

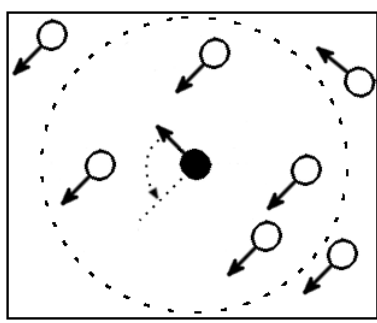

(b) $F_{a}$

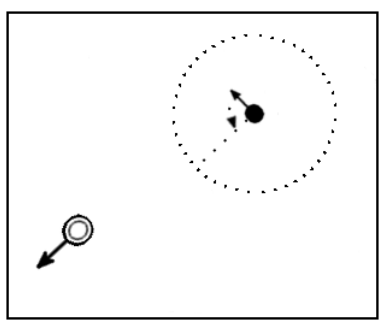

(d) $F_{a v}$

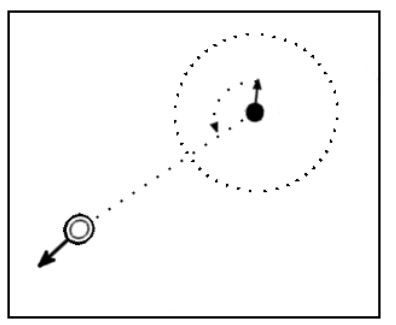

(e) $F_{c v}$

Figure 2 - Interaction rules. The dotted circunferences represent the interaction area of one agent (in black). Do not confuse this area with the VAIR. At (d) and (e) the white agent is the virtual agent.

$F_{a}$ and $F_{c}$ do the same than alignment and cohesion rules defined by Reynolds [3]. Each isolated force produce an effect but we are interested on combinations of them. For this case the resulting force $F$ is calculated as follows in $\mathrm{Eq} 3$.

$$
F=\frac{\alpha_{a} F_{a}+\alpha_{c} F_{c}+\alpha_{a v} F_{a v}+\alpha_{c v} F_{c v}}{\alpha_{a}+\alpha_{c}+\alpha_{a v}+\alpha_{c v}}
$$

in which the $\alpha_{a}, \alpha_{c}, \alpha_{a v}, \alpha_{c v}$ are control coefficients and each force is unitary. The separation force does not appear here because it is used isolated when a collision between two individuals is about to happen.

The virtual agent represents the desired trajectory for the parallel formation, i. e., the position over time that you want your formation to follow. 


\section{RESULTS}

We tested two approaches to calculate the virtual forces. The fist one (S-1) does it just like Reynolds [3], considering all the agent neighbors equally (Figure $3 \mathrm{a}$ ). The second (S2) takes into account the distances between the agent and its neighbors through perception zones (Figure 3b). In this case, when a neighbor is in the first radius, closer to the agent, only $F_{s}$ is calculated. Following the same logic, when the neighbor is in the second zone (ZA), only the force of alignment $F_{a}$ is computed, and in the third zone (ZC), only $F_{c}$ is considered. This approach follows characteristics observed in nature with schools of fish. Some species of fish increase their velocity module when a neighbor is far ahead (attraction), and decrease when it is too close (separation) [4].

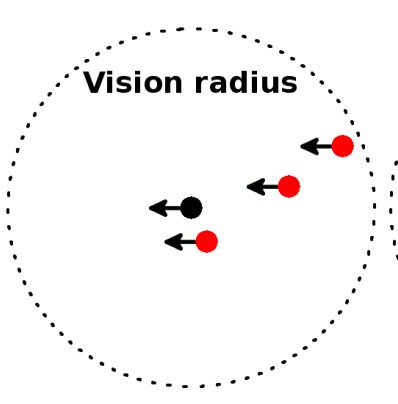

(a) C-1

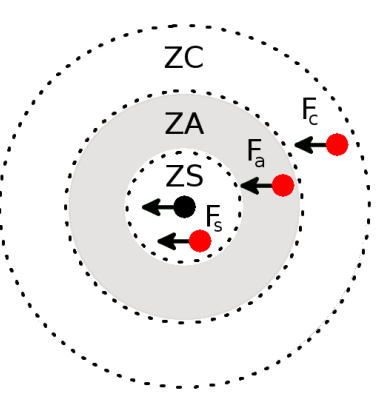

(b) $\mathrm{C}-2$
Figure 3 - Calculation of forces: S-1) Considering the entire agent vision radius equally; and $S-2$ ) Considering zones of perception (ZS - Zone of separation, ZA - Zone of alignment and ZC - Zone of cohesion). The black agent is an ordinary agent of the model and the dotted region corresponds to its vision region (perception region). Agents in red are its neighbors.

We tested four strategies (combinations of forces) as shown in Table 1. The application of forces depends on whether the agent is inside or outside the VAIR.

Table 1 - Combinations of forces (strategies) used when the agents are inside or outside the VAIR.

\begin{tabular}{|c|l|l|}
\hline Strategy & Outside the VAIR & Inside the VAIR \\
\hline S-1.1 (S-2.1) & $F_{a}, F_{c v}$ & $F_{a v}$ \\
\hline S-1.2 (S-2.2) & $F_{c v}$ & $F_{a v}, F_{a}$ \\
\hline S-1.3 (S-2.3) & $F_{c v}, F_{c}$ & $F_{a v}, F_{a}$ \\
\hline S-1.4 (S-2.4) & $F_{c v}, F_{c}, F_{a v}, F_{a}$ & $F_{a v}, F_{a}$ \\
\hline
\end{tabular}

Definition 1 Agents are in a desired formation (DF) if all of them are moving in a parallel formation and have the same velocity module as the virtual agent.

The idea is to see what kind of parallel formations we can get with different combinations of forces (strategies). We defined three quantifiers:

1. Temporal index $(\tau)$ : time units $(t u)$ from the beginning of the simulation until the DF.
2. Angular uniformity index $(\phi)$ : We calculate the relative angles between the position of each agent and the virtual agent. Then we compute the order parameter (Eq 4 [5]) with those angles.

$$
p_{\theta} \doteq \frac{1}{N} \sum_{j=1}^{N} e^{i \theta_{j}}
$$

with $e^{i \theta_{k}}=\cos \theta_{j}+i \sin \theta_{j}$.

In this case, $\phi=\left|p_{\theta}\right|$. When $\phi=0$ the agents are uniformly distributed around the virtual agent. On the other hand, if $\phi=1$ then the agents are at the same position.

3. Radial uniformity index $(\rho)$ : The mean of the distances between each agent and the virtual agent.

We performed experiments with all combinations of $\alpha_{a}$, $\alpha_{c}, \alpha_{a v}, \alpha_{c v} \in\{1,2, \cdots, 10\}$. Each configuration was simulated 10 times because the initial positions and heading angles are random.

Initial conditions:

- Population of 12 agents randomly positioned inside a rectangle of dimensions $36 \times 76 \mathrm{bl}$, as shown in Figure 1.

- Vision radius of each agent: $5.5 \mathrm{bl}$.

- Minimum separation: $2.5 \mathrm{bl}$.

- $F_{s}: 2$ decimal degrees.

- $F_{a}=F_{c}=F_{a v}=F_{c v}: 1$ decimal degree.

- Heading angle: Random.

- $\left|v_{a}\right|=1 \mathrm{bl} / \mathrm{tu},\left|v_{\text {out }}\right|=1.4 \mathrm{bl} / \mathrm{tu}$.

- VAIR: $25 \mathrm{bl}$.

- Virtual agent heading angle: 180 decimal degrees.

- $\alpha_{\text {lim }}: 25 \mathrm{bl}$.

- $\beta_{\text {lim }}: 5$ decimal degrees.

We only consider the cases that reach the DF for all 10 attempts and obeying $\tau \leq 3000 \mathrm{tu}$. Simulations were limited to this temporal limit of $3000 \mathrm{tu}$ due to the large number of combinations. Also, this value was set because the agents usually converge in less than $2000 \mathrm{tu}$, according to experimental observations.

Figure 4 shows the desired formations achieved in terms of the maximum and minimum values of $\tau, \phi$ and $\rho$. 


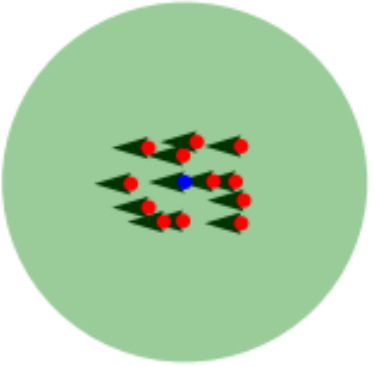

(a) $\min (\tau)=871: \mathrm{S}-2.2$

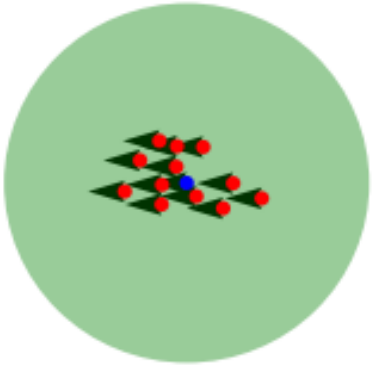

(c) $\min (\phi)=0.09: \mathrm{S}-1.3$

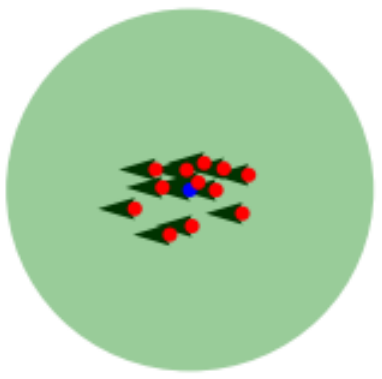

(e) $\min (\rho)=5.20: \mathrm{S}-1.2$

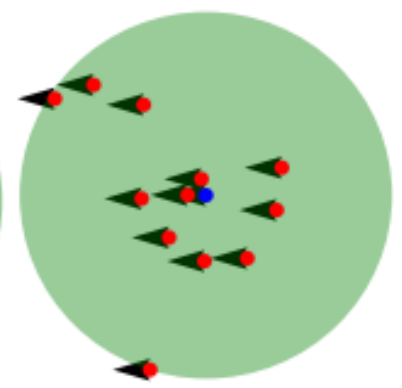

(b) $\max (\tau)=1971: \mathrm{S}-1.1$

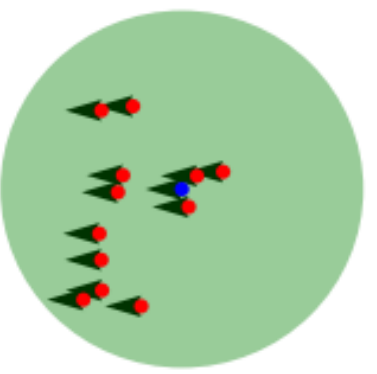

(d) $\max (\phi)=0.36: \mathrm{S}-1.4$

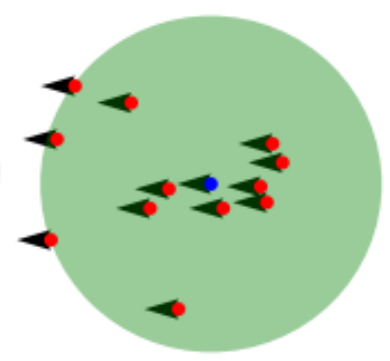

(f) $\max (\rho)=13.88: \mathrm{S}-2.4$
Figure 4 - Desired formations for the tested configurations in terms of the maximum and minimum values achieved for $\tau, \phi$ and $\rho$. Configurations: (a) S-2.2: $\alpha_{a}=8, \alpha_{a v}=9$ and $\alpha_{c v}=4$; (b) S-1.1: $\alpha_{a}=7, \alpha_{a v}=1$ and $\alpha_{c v}=3$; (c) S-1.3: $\alpha_{a}=1, \alpha_{c}=$ 4, $\alpha_{a v}=4$ and $\alpha_{c v}=9$; (d) S-1.4: $\alpha_{a}=3, \alpha_{c}=5, \alpha_{a v}=7$ and $\alpha_{c v}=9$; (e) S-1.2: $\alpha_{a}=3, \alpha_{a v}=10$ and $\alpha_{c v}=10$; (f) S-2.4: $\alpha_{a}=2, \alpha_{c}=3, \alpha_{a v}=6$ and $\alpha_{c v}=9$.

\section{DISCUSSION}

If we want the agents to converge as fast as possible, a good choice would be strategy S-2.2 with the configuration of Figure 4a. Otherwise, if the aim is to get them very close to each other the best option is the configuration of strategy S-1.2 of Figure 4e. The resulting formations depends on both the chosen strategy and configuration. In this sense, some strategies are more suitable for some objectives than others.

In a situation in which we want the agents to spread uniformly inside the VAIR, $\phi$ would be near zero and $\rho$ around the half of VAIR size. This is one example of what can be done in terms of search in parameter space.

\section{CONCLUSION}

We presented a reactive model whose aim is to make agents converge to parallel formations heading to desired directions. We introduced the so called virtual agent whose role is to indicate the direction of motion to the formations and also to evaluate the results. The agents interact via five rules depending on a chosen strategy (combination of rules) and a configuration (weight of each rule).

As a future work we will do a stability analysis and also perform experiments with actual robots. Also, considering that agents have sensory limitations, we can use this model as a pre-step before turning to another model with more controls, since its principle is to group agents into a bounded region.

\section{ACKNOWLEDGMENTS}

We would like to thank the Conselho Nacional de Desenvolvimento Científico e Tecnológico - CNPq, and the Coordenação de Aperfeiçoamento de Pessoal de Nível Superior CAPES, for the financial support. EENM thanks FAPESP, process 2011/50151-0, for its support.

\section{References}

[1] T. Vicsek and A. Zafeiris, "Collective motion," Physics Reports, vol. 517, no. 3-4, pp. 71 - 140, 2012. Collective motion.

[2] D. Paley, N. E. Leonard, R. Sepulchre, D. Grünbaum, and J. K. Parrish, "Oscillator models and collective motion: Spatial patterns in the dynamics of engineered and biological networks," IEEE Control Systems Magazine, vol. 27, no. 4, pp. 89-105, 2007.

[3] C. W. Reynolds, "Flocks, herds and schools: A distributed behavioral model," SIGGRAPH Comput. Graph., vol. 21, pp. 25-34, Aug. 1987.

[4] Y. Katz, K. Tunstrom, C. C. Ioannou, C. Huepe, and I. D. Couzin, "Inferring the structure and dynamics of interactions in schooling fish," Proceedings of the National Academy of Sciences, vol. 108, no. 46, pp. 18720-18725, 2011.

[5] S. H. Strogatz, "From kuramoto to crawford: Exploring the onset of synchronization in populations of coupled oscillators," Phys. D, vol. 143, pp. 1-20, Sept. 2000. 